\title{
Die akademische Forschung trägt eine ethische Verantwortung
}

Aus Sicht von Prof. Lars Zender ist Drug

Repurposing eine Kompomente für die Entwicklung neuer Medikamente. Eine weitere ist akademische Forschung.

Prof. Dr. med. Lars Zender

Leiter der Klinik für Innere Medizin VIII -

Klinische Tumorbiologie am

Universitätsklinikum Tübingen scher Einrichtungen?
? Warum ist die Forschung zum Drug Repurposing eher eine Domäne kleiner Firmen und akademi-

Prof. Lars Zender: Die Begeisterung der großen Pharmafirmen hält sich tatsächlich in Grenzen, was in erster Linie mit den begrenzten Gewinnaussichten zu tun hat. Denn ist die Entwicklung des wiederentdeckten Moleküls schon einige Zeit her, ist die Restlaufzeit des Patentschutzes entsprechend verkürzt. Das Interesse, wiederentdeckte Präparate in GMP ("good manufacturing practice")-Qualität herzustellen oder gar klinische Studien zu bezahlen, sinkt somit beinahe gegen Null. Es braucht daher unbedingt vernünftige Lösungsansätze, wie künftig vielversprechende Wirkstoffe und Moleküle zu einem realistischen Preis ausgelöst und in der akademischen Forschung weiterentwickelt werden können, um sie schließlich möglichst kostengünstig dem Patienten anbieten zu können. Die akademi- sche Forschung trägt hier eine ethische Verantwortung.

? Welche Zukunft prophezeien sie der Methode des Drug Repurposing?

Zender: Keine Frage, das Drug Repurposing wird ein wichtiger Aspekt bei der Entwicklung neuer Medikamente sein. Aber es ist - wie gesagt - eben nur eine Komponente. Darüber hinaus entscheidend wird der Ausbau der Academic-Drug-Discovering-Strukturen sein. Schon heute kommen mehr als $60 \%$ der innovativen onkologischen Präparate aus der akademischen Forschung, die großen Unternehmen liefern gerade mal noch $40 \%$. Viele Pharmaunternehmen haben ihre eigenen Entwicklungsabteilungen verkleinert und suchen frühzeitig die Kollaboration mit akademischen Einrichtungen. Das ist weiter auszubauen. In Deutschland hinken wir der Entwicklung leider sehr hinterher. Tübingen ist eines von gerade einmal drei Instituten in Deutschland, die Mitglied im weltweit agierenden Academic Drug Discovering Consortium sind. In den USA sind es über 150 Zentren.

! Herr Prof. Zender, wir danken für das Gespräch! heit, Neben- und Wechselwirkungen zurückgreifen, und somit kritische Phasen der Wirkstoffentwicklung wie etwa die Phase „präklinische Sicherheit, Pharmakologie und Toxikologie", die nicht ohne Grund als „valley of death“ bezeichnet wird, überspringen, so die Hoffnung.

\section{Welche Probleme es zu lösen gilt}

Alte Wirkstoffe neu zu positionieren, bleibt nichtsdestotrotz eine gewaltige Aufgabe. Neben den zugelassenen Medikamenten dürfe man die zahlreichen Wirkstoffmoleküle nicht außer Acht lassen, die es aus den unterschiedlichsten Gründen nicht bis zur $\mathrm{Zu}$ lassung geschafft haben. Auch diese bergen ein riesiges Potenzial, nicht zuletzt vor dem Hintergrund der individualisierten Krebstherapie, wie sie etwa durch das Tübinger Center for Academic Drug Discovery \& Development (TÜCAD2) und das Zentrum für Personalisierte Medizin (ZPM) der Uniklinik Tübingen vorangetrieben werden, wie Zender erklärt.

Doch selbst wenn sich schätzungsweise für $75 \%$ aller Wirkstoffe neue Anwendungsgebiete theoretisch finden lassen könnten [Nosengo N. Nature. 2016;534(7607):314-6], tatsächlich zur Anwendung kommt schlussendlich nur ein Bruchteil. Voraussichtlich $68 \%$ der Wirkstoffe werden bereits in der Phase II durchfallen und fast die Hälfte der verbleibenden Kandidaten in Phase III, so die Schätzung [Nosengo N. Nature. 2016;534(7607):314-6].

Daher muss bei der Kandidatenauswahl exakt auf die präklinischen Modelle geachtet werden, in denen die Daten erhoben worden sind, wie Zender betont. Teste man etwa den beim Leberkrebs als Standardtherapeutikum geltenden Proteinkinaseinhibitor Sorafenib an menschlichen Tumorzellen, die in immundefizienten Mäusen gewachsen sind, erhalte man vollkommen unrealistische Therapieantworten. Erst in ausgefeilteren präklinischen Modellen, in denen auch die Resistenzentwicklung menschlicher $\mathrm{Tu}$ moren berücksichtigt wird, erreicht man realistische Ergebnisse. Im Falle von Sorafenib einen Überlebensvorteil von etwa drei Monaten beim Menschen.

\section{Schritt für Schritt zum Ziel}

Um ein geeignetes Wirkstoffmolekül herauszufiltern, gibt es im Prinzip zwei Ansätze. Ein Weg führt über sogenannte Substanzbibliotheken, in denen zugelassene und ehemals zugelassene Substanzen enthalten 